\title{
In Situ Coupling Applied Voltage and Synchrotron Radiation: Operando Characterization of Transistors
}

\author{
Anton Davydok', Yuriy N. Luponosov², Sergey A. Ponomarenko ${ }^{2}$ and Souren Grigorian ${ }^{3,4^{*}}$ (D)
}

\begin{abstract}
A compact voltage application setup has been developed for in situ electrical testing of organic field effect transistors in combination with X-ray scattering studies at a synchrotron beamlines. Challenges faced during real condition in-operando test of newly developed OFETs originated an idea of creation of a new setup which excludes number of factors that make experiments complicated. The application of the setup is demonstrated on a prototype of an organic transistors based on $a, \omega$-dihexyl-a-quaterthiophene molecules. The new setup allows to monitor material structural changes by $X$-ray scattering under applied voltage conditions and their direct correlations. The versatile setup eliminates possible shadowing effects and short circuits due to misalignment of the contacts. The electrical stability of the prototypes was characterized by the application of different voltage values. Corresponding structural changes were monitored by grazing $X$-ray scattering technique before, during and after the voltage was applied. The selected oligothiophene material with proved transistor properties shows high stability and directional anisotropy under applied voltage conditions. Thanks to a compact and flexible design of the setup, different type of small dimension devices could be studied under external voltage conditions at various synchrotron beamlines.
\end{abstract}

Keywords: OFETs, Operando studies, nanoGIWAXS, nanoGIXD, $a, \omega$-dihexyl-a-quaterthiophene

\section{Introduction}

During last decades, organic conjugated material-based devices demonstrate huge potential for new generation of low-cost electronics. Recent reviews report on the semiconducting organic materials of different composition with outstanding electrical and optical properties for applications as functional materials in organic light emitting diodes [1], organic filed-effect transistors (OFETs) [2-4] or organic solar cells $[5,6]$.

Important role in the studies, before application in mass production, is playing a strong connection between investigated material properties and its microstructure and molecular ordering. Molecule with similar

\footnotetext{
*Correspondence: grigorian@physik.uni-siegen.de

${ }^{3}$ Department of Physics, University of Siegen, Walter-Flex-Straße 3,

57072 Siegen, Germany

Full list of author information is available at the end of the article
}

components but with different structure, ordering, distribution, and various bents might demonstrate very different properties and reaction to externally applied conditions $[7,8]$. Rather recent studies shown relevance of the static and dynamic parameters in the electrical performances of OFETs and showed that the radiofrequency model established in a solid-state, it allows a reliable prediction of the performances of organic prototype. Authors are reporting on the effective miniaturization of OFETs, which is expected to provide a useful design guideline also in the integration of organic digital and analog circuits [9]. A lower limit of the mobility of 'highperformance' organic transistors has been established in the range of $1-10 \mathrm{~cm}^{2} \mathrm{~V}^{-1} \mathrm{~s}^{-1}$ [10]. However, this limit could be reached by rather short list of the organic materials such as single crystals or highly ordered films. Small molecules including oligothiophenes are promising candidates for organic electronics applications with 
mobilities up to $1 \mathrm{~cm}^{2} \mathrm{~V}^{-1} \mathrm{~s}^{-1}$ [11] and power conversion efficiency reaching of 10\% [12]. Among different linear oligothiophenes, $\alpha, \alpha$-dihexyl-quaterthiophene (DH4T) is well-known oligothiophenes revealing high field-effect mobility and well-ordered microstructure [13, 14]. In particular, a highly crystalline phase of DH4T with inplane $\pi-\pi$ orientation is beneficial for charge transport [15].

The unique properties of organic semiconductors, issues, and problems being non-typical for inorganic transistors are described in [13]. Important place in this research is taken by in situ experiments, in particular by in situ investigation using X-ray diffraction and scattering techniques [16-18]. Since most of the smart devices are designed as layered systems on substrate including electrodes and deposited organic material, an instrument sensitive to surfaces and interfaces is necessary. The technique of grazing X-ray scattering/diffraction with controllable penetration depth is well-established for characterization of organic semiconductors and their properties. Grazing incidence small and wide angle scattering (GISAXS, GIWAXS) as well as grazing incidence $\mathrm{X}$-ray diffraction (GIXD) are widely used methods for functional layers characterization of organic electronics device prototypes [19]. Benefits of the technique already have been demonstrated in various studies of the material structure for organic electronics. In situ investigations of organic transistors were performed and reported based on absorption spectroscopy [20,21], X-ray diffraction experiments [22, 23] or combination of both [4]. A structural stability of 5,5'-bis(naphth-2-yl)-2,2'-bithiophene $\left(\mathrm{NaT}_{2}\right)$-based OFETs was studied during operando experiment in combination with GIXD technique. A device with $500-\mu \mathrm{m}$-long channel was tested by submillimeter X-ray beam. Rather detail analysis of the material structure demonstrated a high structural stability with variation of $1 \%$ in range of up to $40 \mathrm{~V}$ [24]. Strong contribution to pentacene-based OFETs was demonstrated from another operando experiment combing with X-ray diffraction [25]. In this work, nanometer-sized monolayers system response on external applied voltage was monitored by XRD. The studies demonstrated slight tilt and reorganization of the molecules at grain boundaries, under applied voltage molecules forming highly crystalline and textured crystal domains.

The structural evolution of the polymer: fullerene active layers during the drying process give insight of the microstructure formation linked to the optimization of the solar cells [26]. Moreover, the solution processing and the microstructure formation can be coupled with electrical response of the active layers [27, 28]. Usually scattering X-ray signal is rather weak in solution phase and at the initial stage of the microstructure formation and such studies would be impossible to realize without usage of synchrotron radiation.

Furthermore, the synchrotron beam focused to submicron or smaller size becomes a necessary tool to achieve a sufficiently high spatial resolution and strong enough signal to monitor microstructural changes during the instrument operation. Typical synchrotron beamlines that deliver a nanometer beam are very spatially limited, what requires from the experimental setup to be compact and flexible. During such investigations, one should consider a number of limiting factors, except abovementioned, which are radiation damage, complicity of contacts alignment, small size of the systems. Importance and complicity of operando synchrotron measurements with nanosize beam were shown in [7] where potential of nanoGIXD experiment was used for a study of real-sized organic field effect transistors under applied voltage.

However, there is no routine way for detailed studies of the material behavior under real working conditions. Particular difficulties are related to the small dimensions of the active layers of the modern smart devices and circuits.

In this paper, we present a self-developed setup for in situ studies under applied voltage, which is compact and compatible with different synchrotron beamlines. The voltage in situ setup for Application at Synchrotron beamlines (VINAS) is a new tool dedicated for operando studies of the multilayer-based system that supposed to be operated under external voltage conditions. A selfexplanatory and easy-to-use setup simplifies the electrical connection and eliminates any blocking of an X-ray beam. VINAS can be operated under broad ranges of applied voltages, which make the setup helpful for studies of almost all types of new vertical microelectronic devices. The potential of the setup is demonstrated on operando studies of working prototype OFET device based on DH4T oligothiophene. Results obtained during the reported experiment complete the knowledge of the device stability under externally controlled experimental conditions.

\section{Methods}

\section{Setup Description}

The voltage in situ setup for application at synchrotron beamlines is a compact device compatible with various synchrotron beamlines for in situ studies of a multilayers system with promising electrical properties. The VINAS setup is self-developed device designed and constructed by Soft matter physics group (University of Siegen, Germany). The potential of the setup was explored by operando studies of the OFETs devices based on DH4T. Circuit board with the sets of 20 OFET devices was fixed by special plastic screws on the board corners in harmless 


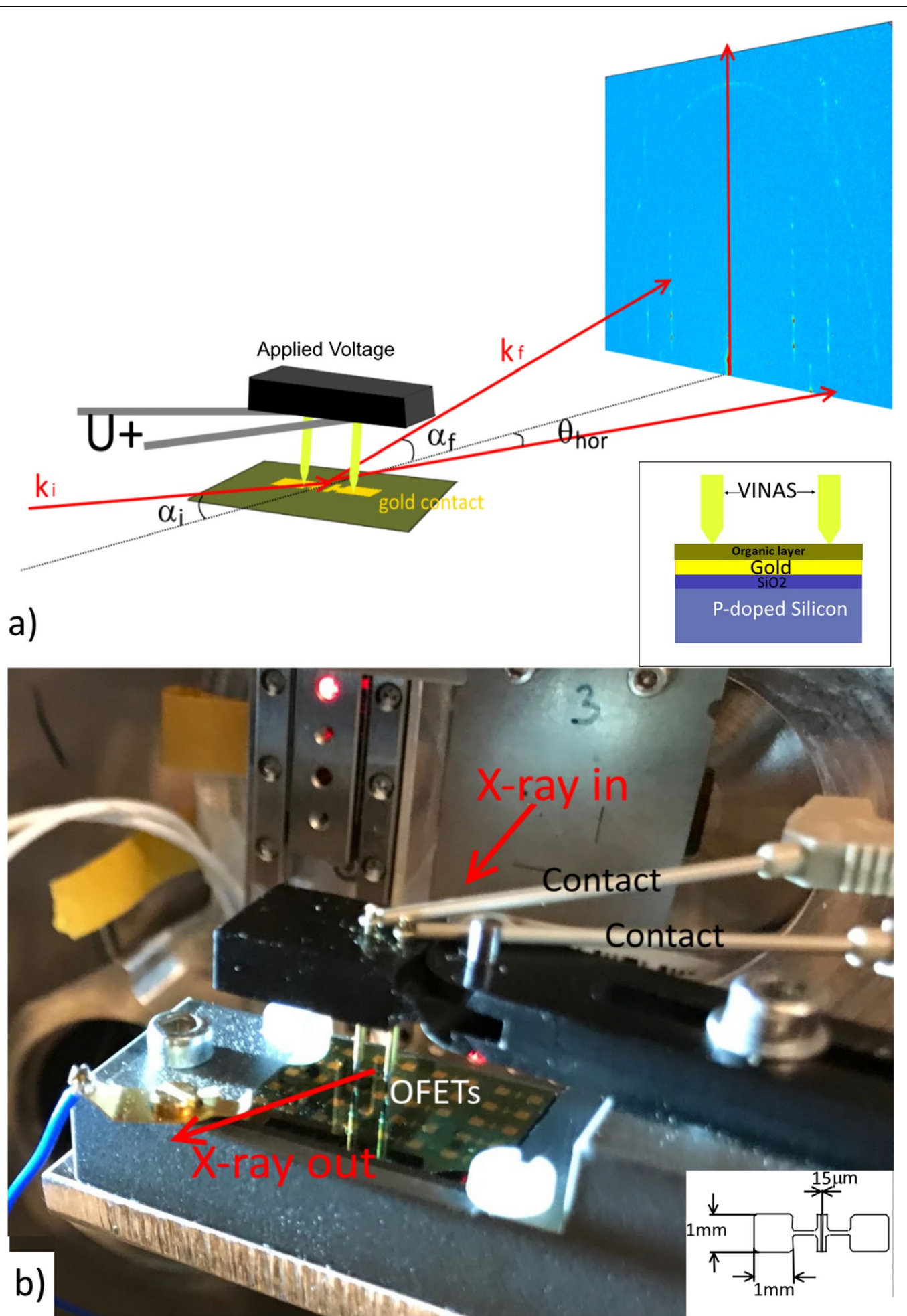

Fig. 1 a Scheme of the VINAS setup with installed sample and marked vertical contacts during in situ measurements; b photograph of VINAS setup installed at P03 nanofocus endstation (PETRA III, DESY), real setup of reported studies, inset: schematic image of the OFET device 
way. OFETs themselves are multilayer system (see schematical inset in Fig. 1a) consisting of a silicon substrate, followed by a 300-nm-thick silicon oxide layer with thermally evaporated gold electrodes on top of it. Finally, a $100 \mathrm{~nm}$ DH4T thin layer was thermally evaporated making bottom source-drain, bottom gate device. For sake of simplification, the gold electrodes are shown uncovered in Fig. 1a. Special probing setup enables the voltage to be applied to any of the selected transistor by vertical 7-mmlong electrodes leaving free space for the incoming and scattered X-ray beam (see Fig. 1). A custom-made setup enables local positioning of all 20 different single-channel OFETs using special gold-sputtered pins with springs to connect to the contact pads of the single-channel OFETs in the bottom contact geometry. For operando studies, we have chosen central OFETs with the channel length of $15 \mu \mathrm{m}$ and channel width of $1 \mathrm{~mm}$.

\section{X-ray Characterization}

The reported experiment was performed at the Nanofocus Endstation of P03 beamline at PETRA III Synchrotron, DESY (Hamburg, Germany) [29]. The VINAS setup (Fig. 1b) was installed on a hexapod from SmarAct that allows scanning along three orthogonal axes and corresponding angles. Monochromatic synchrotron beam with photon energy of $16.98 \mathrm{keV}$ was focused down to size of $300 \times 300 \mathrm{~nm}^{2}$ by means of Kirkpatrick-Baez mirrors. The measurements were performed in GIWAXS geometry with the angle of incidence $\alpha_{i}=0.045^{\circ}$. Such shallow angle resulted in footprint of around 400 um which enables to enhance scattering signal and to measure microstructure along main part of the active channel (with the width of $1 \mathrm{~mm}$ ). Scattered signal was recorded by CCD type Photonic Science area detector with pixel size of $62 \times 62 \mu \mathrm{m}^{2}$ and dimensions of $2940 \times 2940$ pixels $^{2}$ installed on the distance of $248.9 \mathrm{~mm}$ from the sample position.

\section{Data Analysis}

The data analysis performed with the use of DPDAK software [30] and self-written codes on MATLAB. All peak parameters (as peak position, FWHM, peak area) were obtained through the fitting procedure with Gaussian function.

\section{Sample Preparation}

The OFET testbeds with 20 different devices on a single chip were purchased from Ossila Limited, Sheffield, United Kingdom. The DH4T thin layer was thermally evaporated in a vacuum deposition chamber under high vacuum at $10^{-6} \mathrm{mbar}$ with the evaporation rate of $0.2 \AA / \mathrm{s}$ at room temperature.

\section{Materials Synthesis and Characterization}

D4HT was synthesized through oxidative coupling reaction of lithium derivative of $5^{\prime}$-hexyl-2,2'-bithienyl-5-yl at the presence of $\mathrm{CuCl} 2$ (See Fig. 2a), similar to the method described previously [31]. A solution of lithium diisopropylamide (LDA; $1.61 \mathrm{ml}$ of $2 \mathrm{M}$ solution in THF/heptane/ ethylbenzene, $3.2 \mathrm{mmol}$ ) was added dropwise to the solution of 5-Hexyl-2,2'- bithiophene $(0.97 \mathrm{~g}, 3.9 \mathrm{mmol})$ in anhydrous THF $(15 \mathrm{ml})$ at $-78{ }^{\circ} \mathrm{C}$ under nitrogen. It was

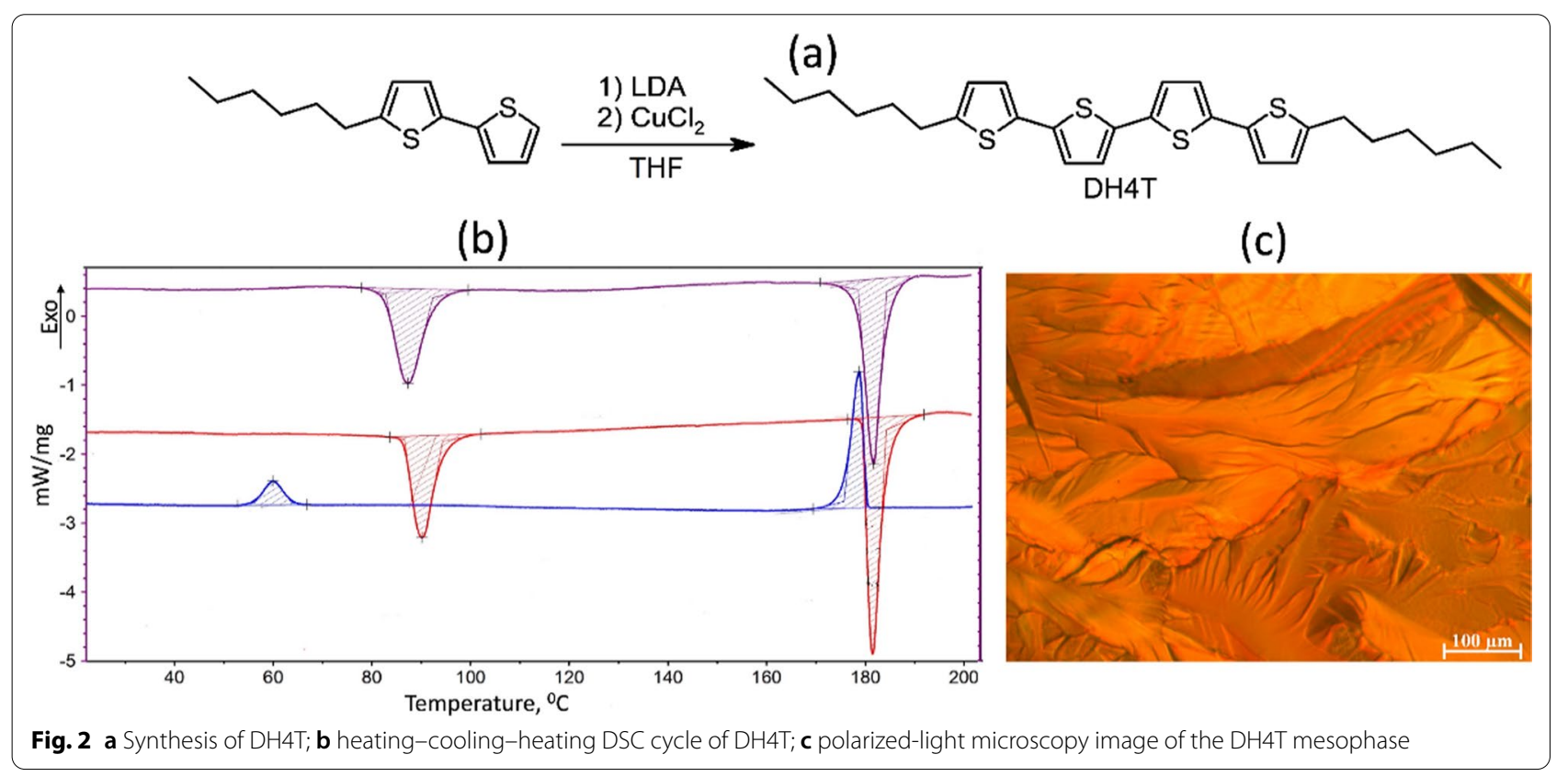


stirred for $30 \mathrm{~min}$ at $278{ }^{\circ} \mathrm{C}$, and anhydrous powdered $\mathrm{CuCl} 2$ (440 mg, $3.3 \mathrm{mmol}$ ) was added in one portion to the white-yellowish suspension, upon which the color of the solution changed to a black. The mixture was stirred until it returned to room temperature and then for an additional $60 \mathrm{~min}$ at $30{ }^{\circ} \mathrm{C}$. The mixture was poured into $200 \mathrm{ml}$ of water, containing $10 \mathrm{ml}$ of $1 \mathrm{M}$ hydrochloric acid. Then, $300 \mathrm{ml}$ of diethyl ether was added and a yellow solid formed in the ether phase, which was washed three times with $200 \mathrm{ml}$ of water and filtered to give a yellow precipitate, which was washed with diethyl ether and dried under vacuum to give. The crude product was purified by passing through silica gel column (eluent-warm toluene) followed by recrystallization from toluene/hexane mixture to give $500 \mathrm{mg}(63 \%)$ of yellow crystals. Chemical structure and purity of D4HT were proven by ${ }^{1} \mathrm{H}$ NMR spectroscopy and elemental analysis. ${ }^{1} \mathrm{H}$ NMR $(250 \mathrm{MHz}, \mathrm{CDCl} 3$, TMS/ ppm): 0.89 (t, $6 \mathrm{H}, J=6.7 \mathrm{~Hz}$ ), $1.23-1.45$ (overlapped peaks, $12 \mathrm{H}), 1.67(\mathrm{~m}, 4 \mathrm{H}), 2.78(\mathrm{t}, 4 \mathrm{H}, J=7.3 \mathrm{~Hz}), 6.67(\mathrm{~d}, 2 \mathrm{H}$, $J=3.7 \mathrm{~Hz}), 6.96(\mathrm{~d}, 2 \mathrm{H}, J=3.4 \mathrm{~Hz}), 6.99(\mathrm{~d}, 2 \mathrm{H}, J=3.7 \mathrm{~Hz})$, 7.01 (d, 2H, J=3.7 Hz). Calc.for C28H34S4: C, 67.42; H, 6.87; S, 25.71. Found: C, 67.31; H,6.91; S, 25.66\%.

Differential scanning calorimetry (DSC) scans were obtained with PerkinElmer DSC7 system with $10^{\circ} \mathrm{C} / \mathrm{min}$ heating rate in temperature range of $30-200{ }^{\circ} \mathrm{C}$ for all compounds (Fig. 2c). Nitrogen flow of $50 \mathrm{~mL} / \mathrm{min}$ was used. Optical polarizing microscopy was performed on Axioscop $40 \mathrm{~A}$ Pol, Carl Zeiss (Germany) equipped with a heating module.

\section{Results and Discussion}

The VINAS setup (Fig. 1) was used for in-operando test of organic transistor prototype based on DH4T being one of the well-known and promising p-type organic semiconducting materials for further application in organic electronics. Before the reported studies, crystallinity of the organic layer was characterized by DSC heating scans. These scans of DH4T exhibit two peaks at $87{ }^{\circ} \mathrm{C}$ and $182^{\circ} \mathrm{C}$, corresponding to the first-order transitions (Fig. 2a) with enthalpies of 36 and 63 Jg-1, respectively. As-received the material in a crystalline phase [32], which changes to a smectic mesophase above $87^{\circ} \mathrm{C}$ (Fig. 2b) [33]. Sample with a crystalline layer of $100 \mathrm{~nm}$ thickness was used as basis for the OFET prototype manufacture. The device was prepared with a length of the transistor active channel of $15 \mu \mathrm{m}$. In addition, electrical response of the selected device was obtained proving possible OFETs characteristics of the device. The sample was placed to VINAS setup installed at
P03 nanofocus endstation of PETRAIII synchrotron. Figure 1a exhibits a scheme of X-ray beam scattering from the sample plate toward detector plane. VINAS vertical contacts were placed on gold electrodes of the transistor, and all system was aligned according the beam position by means of an optical microscope directed along the beam provided by the beamline. Focal plane of the microscope is aligned with the focal plane of KB-mirrors ensuring sample alignment according the focused beam position. Photograph of the experimental setup is shown in Fig. 1b demonstrating a sample positioning and its electrical connection regarding synchrotron beam. An alignment procedure of the sample with already installed vertical contacts to the device electrodes consisted of iterative scanning of height, incident angle and tilt of the sample. Nanobeam is extremely sensitive to any minimal misalignment of the system, so we performed the alignments at two different positions of device at the boarder of the channel and in the middle. All found positions where defined as zeros. Incident angle was chosen $\alpha_{i}=0.045^{\circ}$ smaller than the critical angle of $\mathrm{Si}$ substrate at the operated photon energy. Selected incident angle enables to reduce an exposition time and to minimize radiation damage. We scanned along the DH4T transistor channel (Fig. 1b inset) with step of $300 \mathrm{~nm}$ (lateral beamsize) and exposure time of $5 \mathrm{~s} /$ point-a compromise between a good scattering signal and radiation damage. Radiation damage was measured as integrated intensity signal for prolonged time in the same position of the active channel. These intensity measurements reveal microstructural stability during $3000 \mathrm{~s}$. Prior of operando test, an optimal exposure time was chosen and preliminary lateral scan along the channel was performed. Initial structure of the oligothiophene layer with beamsize resolution was obtained for further comparison. Typical scattering pattern measured at the starting point of the scan at the beginning of transistor channel shown in Fig. 3a, b demonstrates the scattering pattern measured at the same position after inoperando test, when applied voltage was removed. On the both patterns are clearly visible three strong vertical rods of $11 \mathrm{~L}, 02 \mathrm{~L}$ and $12 \mathrm{~L}$ families containing number of intense $\mathrm{X}$-ray diffraction peaks and exhibiting highly oriented and well-ordered structure which corresponds to the crystalline phase revealed by DSC. This ordered crystalline phase of DH4T can be described by monoclinic unit cell with the following parameters: $a=6.0 \AA, b=7.8 \AA, c=28.5 \AA$, and $\beta=93.0^{\circ}$ [15]. Further on we concentrated on the selected areas of two vertical truncation rods attributed to reflections family of $11 \mathrm{~L}$ and $02 \mathrm{~L}$ for lower and higher

(See figure on next page.)

Fig. 3 a X-ray scattering pattern obtained from the investigated sample before voltage was applied, $X$-ray peaks selected for further analysis in-plane and out-of-plane structure analysis; $\mathbf{b}$ scattering pattern after applied voltage $\mathbf{c}$ in-plane integrated intensity before and after applied voltage; $\mathbf{d}$ out-of-plane integrated intensity before and after applied voltages; e strain evolution along the tested transistor before and after applied voltages 

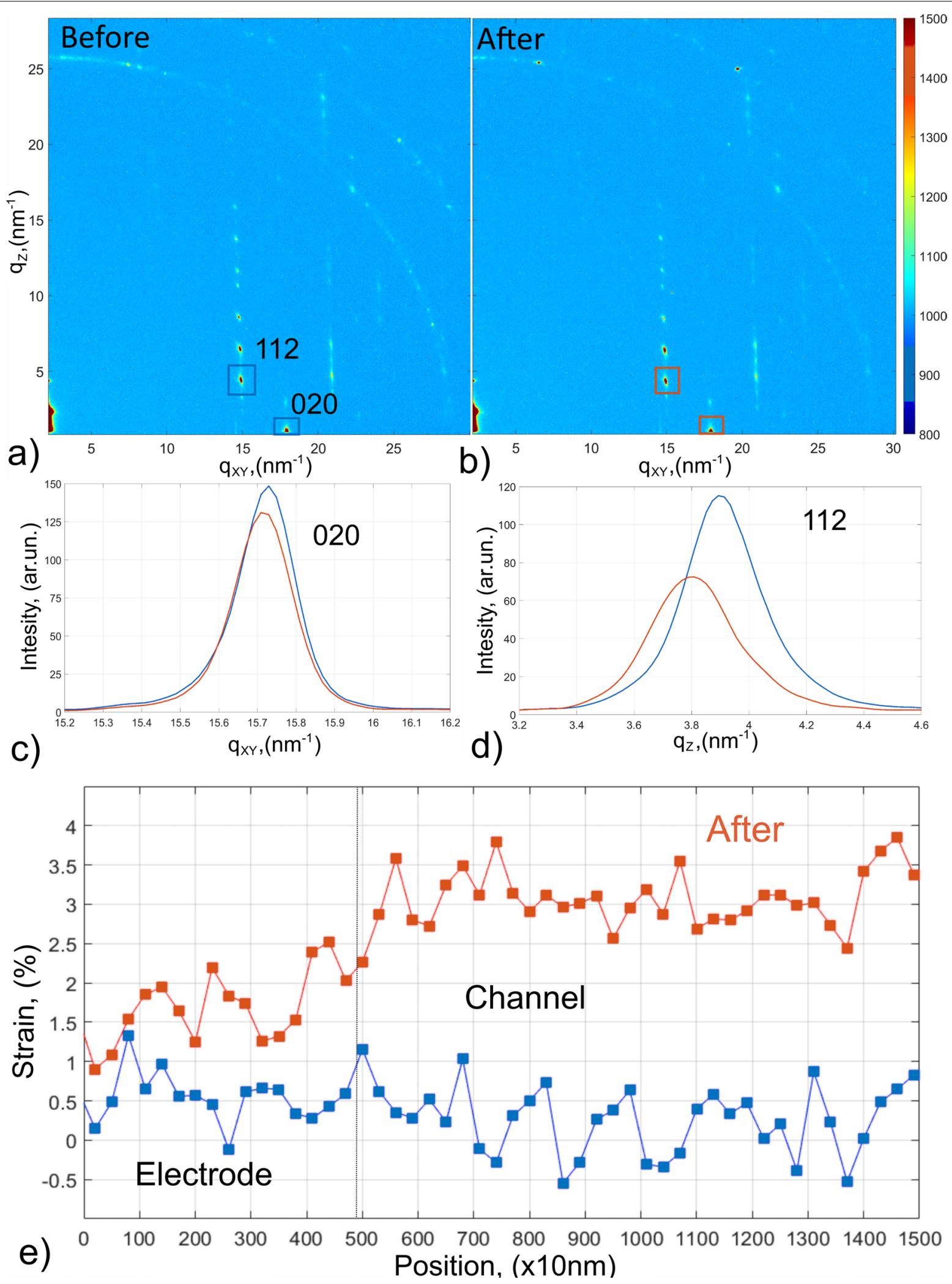

Fig. 3 (See legend on previous page.) 
$q_{x y}$ position correspondingly (Fig. 3a, b). Average distance between the peaks in vertical direction for the $11 \mathrm{~L}$ rod at $q_{x y}=13.2 \mathrm{~nm}^{-1}$ corresponds to $2.9 \mathrm{~nm}$ in real space, what is in a good agreement with vertical stacking distances between molecules. No difference in the average peaks distance is observed comparing diffraction patterns before and after the test (Fig. 3a, b). In addition, two ring-like signals at higher $q$ values could be observed from the pattern corresponding to $\mathrm{Au} 111$ and 200 reflections, which originated by the gold electrode.

We have selected 020 and 112 reflections to characterize structural changes in the in-plane and out-ofplane directions (marked areas in Fig. 3a). We compared changes of scattering signal at the initial stage (before voltage was applied) and after all measurements (when voltage was removed), considering that irreversible changes might be originated by the prototype operation. The peaks shape and position of the selected Bragg reflections were evaluated by fitting integrated intensity curves employing the Gaussian functions. The line profiles for 020 reflection integrated in in-plane direction are shown in Fig. 3c and do not exhibit major changes in both shape and position remain relatively stable along entire experiment. In the out-of-plane direction (integrated 112 reflections Fig. 3d), stronger changes take place with substantial broadening of the peak and expansion of the interplanar distances. Applied voltage originates lattice expansion in the vertical direction and misorientation of the structure shifting positions from initial almost upright-standing molecules. These findings are supported by peak shifts in direction of smaller $\mathrm{q}$ and from peak broadening after the prototype exploitation. Overall in-plane microstructure with improved $\pi-\pi$ stacking reveals to be more stable than out-of-plane one associated with long axis of small molecules which includes flexible chains. The tendency is clearly demonstrated by the comparison of initial and final measurements in Fig. 3c, d. Interestingly, reorientation of the molecules with various tilt angles depending on applied thermal budget was observed and discussed for DH4T and DH5T oligothiophenes in [15, 22], correspondingly. The lattice expansion originates strain fields that can be evaluated from obtained data with hundreds-of-nanometer resolution. Figure 3e demonstrates spatially resolved strains profiles in out-of-plane direction along the transistor channel with beamsize resolution $(300 \mathrm{~nm})$ before and after applied voltages. Here, strains are calculated as a deference of the spacing between molecules $\left(d-d_{0}\right)$ divided by the initial spacing $\left(d_{0}\right)$. At the first part (from 0 to $500 \mathrm{~nm}$ ) corresponding to the film deposited on the gold electrode, the strain is slightly higher than 0 initially. The area from 500 to $1500 \mathrm{~nm}$ refers to the conducting channel and is initially strain-free. Further, a remarkable difference between two areas on the gold and in conducting channel is observed. The changes of the oligomeric molecules on the gold electrode are weaker showing strain values increase of about $1 \%$. This is not the case for the stage after operando test in the conducting channel area where remaining vertical lattice strains increase up to $3 \%$. Interestingly, local scale GIXD studies of poly(3-hexylthiophene) OFETs revealed also the most pronounced changes in microstructural order the interfacial regions of the gold electrodes and the conductive polymer channel and when an electric field was applied [7]. In current work, we are reporting on in situ results obtained with the selected DH4T organic material under fixed applied voltages for the demonstration of the setup feasibility. The OFET mobilities for oligothiophenes with the linear alkyl groups including DH4T were found to be in the range from 0.0004 to $0.08 \mathrm{~cm}^{2} \mathrm{~V}^{-1} \mathrm{~s}^{-1}[14,21]$. More detailed in situ structural and electrical characterization of oligothiophenes will be reported elsewhere. Figure 4 on a blue axis demonstrates the prototype electrical response on the applied voltage during and after $\mathrm{X}$-ray measurements. The current increases almost linearly from $13.3 \mathrm{nA}$ at $10 \mathrm{~V}$ and reaching $136.2 \mathrm{nA}$ at $50 \mathrm{~V}$. After X-ray scans during $1500 \mathrm{~s}$ at the fixed value of $50 \mathrm{~V}$, the current has lowered of about $30 \%$. Afterward, coming back to the $10 \mathrm{~V}$ system has recovered and shows almost same level of the current (empty blue squares, Fig. 4). The lattice response of DH4T under applied voltages is shown on brown axis of Fig. 4 in terms of the relative displacements of the peaks positions in reciprocal space in both directions in-plane(diamond) and out-of-plane(circle). When the voltage of $10 \mathrm{~V}$ was applied, almost no displacement in the in-plane direction was observed, while at the same time the out-of-plane peak moved in the negative direction for $0.05 \mathrm{~nm}^{-1}$. With increasing voltage to $50 \mathrm{~V}$, we observe shifts in the positive direction for both cases-for out-of-plane $0.1 \mathrm{~nm}^{-1}$ and $0.09 \mathrm{~nm}^{-1}$ for in-plane components in total. After the voltage was completely removed, the in-plane component returned almost reversibly to the initial value-differs only for $0.017 \mathrm{~nm}^{-1}$, the out-of-plane directions were more sensitive again and show differences of $0.155 \mathrm{~nm}^{-1}$ compared to the previous position and $0.096 \mathrm{~nm}^{-1}$ compared to the initial stage. From this, we can conclude that the orientation of the structural blocks-molecules-in-plane direction is more stable than in normal direction to the sample surface, but the stability over all is still better compared to other materials in both directions $[7,22]$. There are no significant changes in the position of in-plane peak and overall trends supports higher stability of in-plane components in comparison with out-of-plane ones.

The results of operando tests on semiconductor polymer OFETs with nanosized synchrotron beam and their 


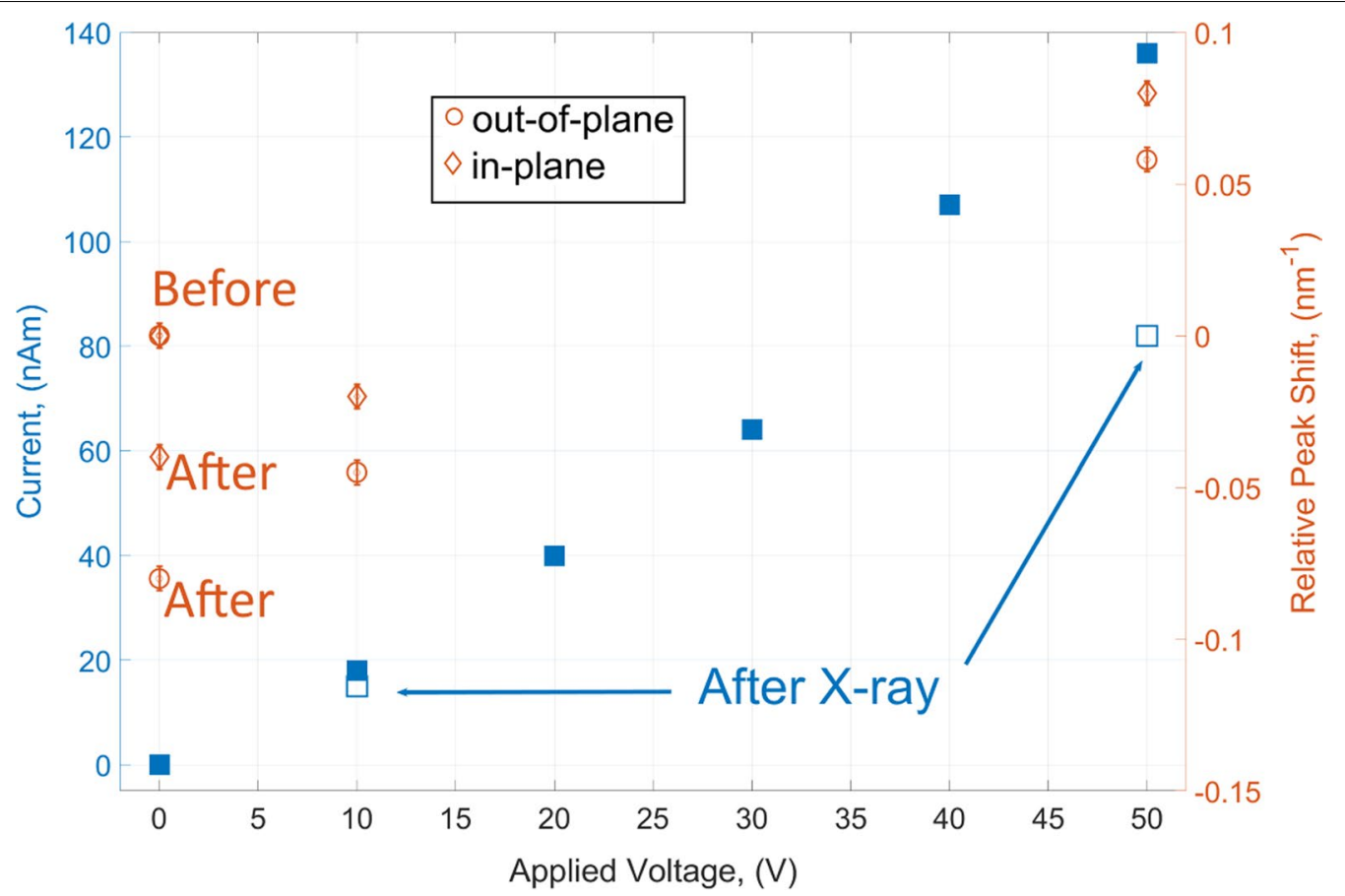

Fig. 4 At the blue axis: current response to the applied voltage during (filled squares) and after(empty squares) X-ray measurements; at brown axis: relative shift of the average peak position in in-plane(diamonds) and out-of-plane(circles) directions

importance for the material studies were reported earlier [7]. Such experiments are faced to a number of technical challenges related to precise positioning of the electrical connectors and restricted volume for the sample environment. The use of VINAS allows to decrease a time required for alignments and electrodes connections with a sample and to provide a stable setup well suitable for in situ investigations. The VINAS has been successfully operated for another type of oligothiophenes during in situ testing (to be published). Despite the weak scattering signal due to X-ray focusing, good quality materials' response could be obtained by increasing the exposure time. Stable in-operando conditions during the measurements are ensured by reported self-developed setup. The chosen experimental conditions enable to perform OFETs stability test and monitor material response on the externally applied voltage. The importance of operando stability tests for novel OFETs devices is clearly underlined by the community, and a development of the universal experimental approach demonstrated here is substantial contribution to the field. For the first time, we have demonstrated that the organic material might be characterized in situ in terms of voltage-induced strains with sub-micron resolution. With increasing of interest in low-costs electronics based on $\pi$-conjugated small molecules and polymers, the studies of materials functionality will be pushed to acceleration and request of universal setups for investigations of the material performances will grow.

\section{Conclusions}

In summary, the in situ voltage setup for synchrotron studies has a great potential for the investigation of layer systems under applied voltage by the surface sensitive synchrotron techniques. It allows direct correlations between an externally applied voltage and the response of materials of interest. We have shown the advantages of in situ experiment where different voltages were applied to monitor simultaneously the microstructure and the electrical response. In comparison with conjugated polymers, the thiophene-based D4HT OFETs reveal to be stable under applied voltage conditions where DH4T oligomer preserves its crystalline phase without transition to mesophase. These stable crystalline phase reveals rather week anisotropy of structural properties with minor changes of in-plane components in comparison with out-of-plane ones. The possible applications of the setup are much wider than in the example given here. Considering the intensive studies on transistors based on organic $\pi$-conjugated molecules and the importance of operando experiments, such in situ instrument is necessary and could be helpful for many scientific applications. Thanks to the compatibility with various synchrotron beamlines and laboratory sources, VINAS allows in situ 


\section{X-ray investigations in combination with the applied voltage of almost any multilayer systems.}

\begin{abstract}
Abbreviations
VINAS: Voltage in situ setup for application at synchrotron beamlines; D4HT: Dihexyl-quarterthiophene; OFET: Organic filed-effect transistors; GISAXS/ GIWAXS: Grazing incident small/wide angle X-ray scattering; GIXD: Grazing incident X-ray diffraction; DSC: Differential scanning calorimetry.
\end{abstract}

\section{Acknowledgements}

The authors acknowledge I. Fratoddi (Sapienza University of Rome), U.Pietsch (University of Siegen, Germany) and C. Krywka (Helmholtz Zentrum Geesthacht, Gemany) for fruitful discussions, DESY for the allocated experiment at P03 beamline. S.G. thanks the Department of Chemistry, Sapienza University of Rome for the visiting professorship.

\section{Authors' contributions}

Investigation was contributed by S.G., A.D.; formal analysis was contributed by A.D.; writing —original draft preparation, was contributed by A.D., S.G.; writing - review and editing, was contributed by Y.L., S.P.; validation, methodology were contributed by S.G.; visualization was contributed by A.D.; resources were contributed by Y.L., S.P.; supervision was contributed by S.G. All authors read and approved the final manuscript.

\section{Funding}

The financial support of DAAD-PROCOPE (Project No. 57211900). The work on synthesis of DH4T was supported by the Russian Science Foundation (grant 19-73-30028). DSC data were obtained using the equipment of Collaborative Access Center 'Center for Polymer Research' of the Enikolopov Institute of Synthetic Polymeric Materials of the Russian Academy of Sciences with the financial support from the Ministry of Science and Higher Education of the Russian Federation (Contract 0071-2021-0005).

\section{Availability of data and materials}

The datasets used and/or analyzed during the current study are available from the corresponding author on reasonable request.

\section{Declarations}

\section{Competing interests}

The authors declare no competing interests.

\section{Author details}

${ }^{1}$ Institute for Materials Physics, Helmholtz-Zentrum Hereon, Notkestr 85, 22607 Hamburg, Germany. ${ }^{2}$ Enikolopov Institute of Synthetic Polymeric Materials of the Russian Academy of Sciences, Profsoyuznaya 70, Moscow, Russia. ${ }^{3}$ Department of Physics, University of Siegen, Walter-Flex-Straße 3, 57072 Siegen, Germany. ${ }^{4}$ Department of Chemistry, Sapienza University of Rome, p.le Aldo Moro 5, 00185 Rome, Italy.

Received: 4 August 2020 Accepted: 16 January 2022

Published online: 02 February 2022

\section{References}

1. Bhatnagar PK (2018) Organic light-emitting diodes-a review. In: Khan Z (eds) Nanomaterials and their applications. Advanced structured materials, 84. Springer, Singapore

2. Quinn JTE, Zhu J, Li X, Wang J, Li Y (2017) Recent progress in the development of n-type organic semiconductors for organic field effect transistors. J Mater Chem C 5(34):8654-8681. https://doi.org/10.1039/c7tc0 1680h

3. Ling H, Liu S, Zheng Z, Yan F (2018) Organic flexible electronics. Small Methods 2(10):1800070. https://doi.org/10.1002/smtd.201800070

4. Lamport ZA, Barth KJ, Lee H, Gann E, Engmann S, Chen H, Jurchescu OD (2018) A simple and robust approach to reducing contact resistance in organic transistors. Nature Commun. https://doi.org/10.1038/ s41467-018-07388-3

5. Yan C, Barlow S, Wang Z, Yan H, Jen AKY, Marder SR, Zhan X (2018) Nonfullerene acceptors for organic solar cells. Nat Rev Mater 3:1-19. https:// doi.org/10.1038/natrevmats.2018.3

6. Yang D, Löhrer FC, Körstgens V, Schreiber A, Bernstorff S, Buriak JM, Müller-Buschbaum P (2019) In-operando study of the effects of solvent additives on the stability of organic solar cells based on PTB7-Th:PC 71 BM. ACS Energy Lett 4(2):464-470. https://doi.org/10.1021/acsenergylett. $8 \mathrm{~b} 02311$

7. Grodd LS, Mikayelyan E, Dane T, Pietsch U, Grigorian S (2020) Local scale structural changes of working OFET devices. Nanoscale 12(4):2434-2438. https://doi.org/10.1039/c9nr07905j

8. Mohanrao R, Hema K, Sureshan KM (2020) Topochemical synthesis of different polymorphs of polymers as a paradigm for tuning properties of polymers. Nat Commun 11(1):1-8. https://doi.org/10.1038/ s41467-020-14733-y

9. Sawada T, Yamamura A, Sasaki M, Takahira M, Okamoto T, Watanabe S, Takeya J (2020) Correlation between the static and dynamicresponses of organic single-crystal field-effect transistors. Nat Commun 11:4839. https://doi.org/10.1038/s41467-020-18616

10. Braga D, Horowitz G (2009) High-performance organic field-effect transistors. Adv Mater 21:1473-1486. https://doi.org/10.1002/adma.200802733

11. Halik M, Klauk H, Zschieschang U, Schmid G, Ponamorenko S, Kirchmeyer S, Weber W (2003) Relationship between molecular structure and electrical performance of oligothiophene organic thin film transistors. Adv Mater 15(11):917-922. https://doi.org/10.1002/adma.200304654

12. Min J, Luponosov YN, Cui C, Kan B, Chen H, Wan X, Chen Y, Ponomarenko SA, Li Y, Brabec CJ (2017) Evaluation of electron donor materials for solution-processed organic solar cells via a novel figure of merit. Adv Energy Mater 7:1700465. https://doi.org/10.1002/aenm.201700465

13. Pérez-Fuster C, Lidón-Roger JV, Contat-Rodrigo L, García-Breijo E (2018) Inexpensive measuring system for the characterization of organic transistors. J Sens. https://doi.org/10.1155/2018/4286894

14. Anokhin DV, Defaux M, Mourran A, Moeller M, Luponosov YN, Borshchev OV, Bakirov AV, Shcherbina MA, Chvalun SN, Meyer-Friedrichsen T, Elschner A, Kirchmeyer S, Ponomarenko SA, Ivanov DA (2012) Effect of molecular structure of $a, a^{\prime}$-Dialkylquaterthiophenes and their organosilicon multipods on ordering, phase behavior, and charge carrier mobility. J Phys Chem C 116(43):22727-22736. https://doi.org/10.1021/jp307054r

15. Mikayelyan E, Grodd L, Ksianzau V, Wesner D, Rodygin Al, Schönherr H, Luponosov YN, Ponomarenko SA, Ivanov DA, Pietsch U, Grigorian S (2019) Phase transitions and formation of a monolayer-type structure in thin oligothiophene films: exploration with a combined in situ X-ray diffraction and electrical measurements. Nanoscale Res Lett. https://doi.org/10. 1186/s11671-019-3009-8

16. Todt J, Krywka C, Zhang ZL, Mayrhofer PH, Keckes J, Bartosik M (2020) Indentation response of a superlattice thin film revealed by in-situ scanning X-ray nanodiffraction. Acta Mater 195:425-432. https://doi.org/10. 1016/j.actamat.2020.05.056

17. Erdely P, Schmoelzer T, Schwaighofer E, Clemens H, Staron P, Stark A, Liss KD, Mayer S (2016) In-situ characterization techniques based on synchrotron radiation and neutrons applied for the development of an engineering intermetallic titanium aluminide alloy. Metals 6(1):1-27. https://doi. org/10.3390/met6010010

18. Güldal NS, Kassar T, Berlinghof M, Unruh T, Brabec CJ (2017) In situ characterization methods for evaluating microstructure formation and drying kinetics of solution-processed organic bulk-heterojunction films. J Mater Res 32(10):1855-1879. https://doi.org/10.1557/jmr.2017.190

19. Smilgies DM (2009) Scherrer grain-size analysis adapted to grazing-incidence scattering with area detectors. J Appl Crystallogr 42(6):1030-1034. https://doi.org/10.1107/S0021889809040126

20. Fu Q, Sarapulova A, Trouillet V, Zhu L, Fauth F, Mangold S, Ehrenberg $H$ (2019) In Operando synchrotron diffraction and in operando X-ray absorption spectroscopy investigations of orthorhombic V2O5 nanowires as cathode materials for Mg-Ion batteries. J Am Chem Soc 141(6):23052315. https://doi.org/10.1021/jacs.8b08998

21. Johannes A, Salomon D, Martinez-Criado G, Glaser M, Lugstein A, Ronning C (2017) In operando x-ray imaging of nanoscale devices: Composition, valence, and internal electrical fields. Sci Adv 3(12):1-7. https://doi. org/10.1126/sciadv.aao4044 
22. Mikayelyan E, Vladimirov I, Wesner D, Grodd L, Rodygin Al, Schönherr H, Grigorian S (2016) Impact of substrate temperature on the structure and electrical performance of vacuum-deposited a, $a^{\prime}$-DH5T oligothiophene thin films. RSC Adv 6(116):115085-115091. https://doi.org/10.1039/c6ra2 4609e

23. Aliouat MY, Escoubas $S$, Benoudia MC, Ksenzov D, Duché $D$, Bènevent $E$, Grigorian S (2020) In-situ measurements of the structure and strain of a $\pi$-conjugated semiconducting polymer under mechanical load. J Appl Phys https://doi.org/10.1063/1.5127226

24. Huss-Hansen MK, Lauritzen AE, Bikondoa O, Torkkeli M, Tavares L, Knaapila M, Kjelstrup-Hansen J (2017) Structural stability of naphthyl end-capped oligothiophenes in organic field-effect transistors measured by grazingincidence X-ray diffraction in operando. Org Electron 49:375-381. https:// doi.org/10.1016/j.orgel.2017.07.012

25. Liscio F, Ferlauto L, Matta M, Pfattner R, Murgia M, Rovira C, Biscarini F (2015) Changes of the molecular structure in organic thin film transistors during operation. J Phys Chem C 119(28):15912-15918. https://doi.org/ 10.1021/acs.jpcc.5b03901

26. Sanyal M, Schmidt-Hansberg B, Klein MFG, Colsmann A, Munuera C, Vorobiev A, Barrena E (2011) In-situ X-ray study of drying-temperature influence on the structural evolution of bulk-heterojunction polymerfullerene solar cells processed by doctor-blading. Adv Energy Mater 1(3):363-367. https://doi.org/10.1002/aenm.201100007

27. Grodd L, Pietsch U, Grigorian S (2012) Direct correlation between electric and structural properties during solidification of poly(3-hexylthiophene) drop-cast films. Macromol Rapid Commun 33(20):1765-1769. https://doi. org/10.1002/marc.201200309

28. Radchenko ES, Anokhin DV, Gerasimov KL, Rodygin Al, Rychkov AA, Shabratova ED, Ivanov DA (2018) Impact of the solubility of organic semiconductors for solution-processable electronics on the structure formation: a real-time study of morphology and electrical properties. Soft Matter 14(13):2560-2566. https://doi.org/10.1039/c7sm02408h

29. Krywka C, Keckes J, Storm S, Buffet A, Roth SV, Döhrmann R, Müller M (2013) Nanodiffraction at MINAXS (P03) beamline of PETRA III. J Phys Conf Ser 425(PART 7). https://doi.org/10.1088/1742-6596/425/7/072021

30. Benecke G, Wagermaier W, Li C, Schwartzkopf M, Flucke G, Hoerth R, Zizak I, Burghammer M, Metwalli E, Müller-Buschbaum P, Trebbin M, Förster S, Paris O, Roth SV, Fratzl P (2014) A customizable software for fast reduction and analysis of large $X$-ray scattering data sets: Applications of the new DPDAK package to small-angle $X$-ray scattering and grazing-incidence small-angle X-ray scattering. J Appl Crystallogr 47(5):1797-1803. https:// doi.org/10.1107/S1600576714019773

31. Ponomarenko S, Kirchmeyer S (2003) Synthesis and thermal behaviour of a, a'-didecyloligothiophenes. J Mater Chem 13(2):197-202. https://doi. org/10.1039/b209176c

32. Katz HE, Lovinger AJ, Laquindanum JG (1998) a, $\omega$-Dihexylquaterthiophene: a second thin film single-crystal organic semiconductor. Chem Mater 10(2):457-459. https://doi.org/10.1021/ cm970627p

33. Amundson KR, Katz HE, Lovinger AJ (2003) Phase behavior of a, $\omega$-dihexyl-a-quaterthiophene and ordering on a textured substrate. Thin Solid Films 426:140-149. https://doi.org/10.1016/S0040-6090(02)01295-6

\section{Publisher's Note}

Springer Nature remains neutral with regard to jurisdictional claims in published maps and institutional affiliations.

\section{Submit your manuscript to a SpringerOpen ${ }^{\circ}$ journal and benefit from:}

- Convenient online submission

- Rigorous peer review

- Open access: articles freely available online

- High visibility within the field

- Retaining the copyright to your article

Submit your next manuscript at $\boldsymbol{\Delta}$ springeropen.com 